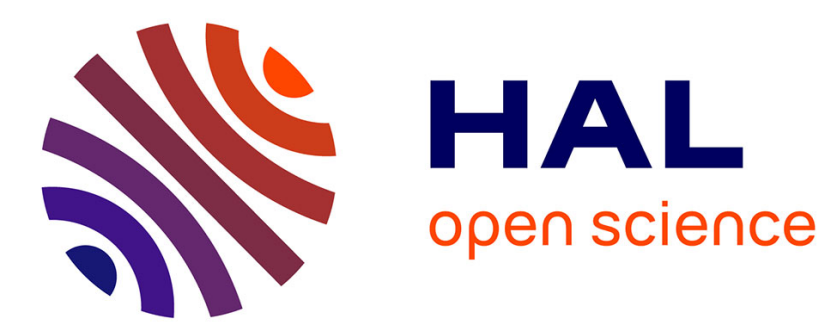

\title{
Optical absorption studies of ion implanted and amorphous silicon
}

U. Zammit, K. Madhusoodanan, M. Marinelli, F. Scudieri, R. Pizzoferrato, F. Mercuri, E. Wendler, W. Wesch

\section{- To cite this version:}

U. Zammit, K. Madhusoodanan, M. Marinelli, F. Scudieri, R. Pizzoferrato, et al.. Optical absorption studies of ion implanted and amorphous silicon. Journal de Physique IV Proceedings, 1994, 04 (C7), pp.C7-113-C7-120. 10.1051/jp4:1994728 . jpa-00253256

\section{HAL Id: jpa-00253256 https://hal.science/jpa-00253256}

Submitted on 1 Jan 1994

HAL is a multi-disciplinary open access archive for the deposit and dissemination of scientific research documents, whether they are published or not. The documents may come from teaching and research institutions in France or abroad, or from public or private research centers.
L'archive ouverte pluridisciplinaire HAL, est destinée au dépôt et à la diffusion de documents scientifiques de niveau recherche, publiés ou non, émanant des établissements d'enseignement et de recherche français ou étrangers, des laboratoires publics ou privés. 


\title{
Optical absorption studies of ion implanted and amorphous silicon
}

\author{
U. Zammit, K.N. Madhusoodanan, M. Marinelli, F. Scudieri, R. Pizzoferrato, F. Mercuri, E. Wendler* \\ and W. Wesch* \\ Dipartimento di Ingegneria Meccanica, $2^{\circ}$ Universita di Roma "Tor Vergata", Via della Ricerca Scientifica, \\ 00173 Rome, Italy \\ * Friedrich-Schiller-Universität Jena, Institut für Feskörperphysik, Max-Wien-Platz 1, 07743 Jena, \\ Germany
}

\begin{abstract}
A detailed study of the implantation induced damage in Si on sapphire, carried out by optical absorption measurements extending from energies above the band gap down to energies far into the sub gap region of $\mathrm{Si}$, is presented. The changes induced in the optical band gap, band edge slopes and in the sub gap features of the spectra are carefully described. The various stages of formation and quenching of divacancies were monitored as a function of implantation conditions and annealing cycles through their 1.8 $\mu \mathrm{m}$ absorption band. It is shown that the divacancies strongly affect the population of band tail states and the annealing studies revealed that the progressive quenching of the divacancy band is followed by the appearance of another absorption band, characteristic of some intrinsic secondary defect, whose annealing behaviour is similar to the one observed for the 5-vacancies EPR spectrum. The study of the structural relaxation process in implanted a-Si gave indications that the process is indeed associated with annihilation of defects as well as average strain reduction in the material, in agreement with earlier indications.
\end{abstract}

\section{Introduction}

Radiation damage in $\mathrm{Si}$ has been studied for the past 30 years. Its interest nowadays is mainly directed towards the understanding and application of the damage accumulated during ion implantation of the material because of its technological implications for devices fabrication and, recently, for "defects engineering" which refers to the controlled introduction of ion induced damage to achieve the desired properties of the material [1]. This is obtained by engineering the interactions between the induced defects and implanted atoms through a suitable choice of the implantation conditions and annealing cycles and therefore derives from a thorough knowledge of damage nucleation and evolution during implantation and subsequent annealing cycles.

In its amorphous structure, implanted $\mathrm{Si}$ has very recently drawn considerable attention because of the mechanisms inducing changes in its physical properties upon annealing, referred to as "structural relaxation". Amorphous $\mathrm{Si}(\mathrm{a}-\mathrm{Si}$ ) is generally viewed as a continuous random network of tetrahedrally coordinated covalently bonded atoms [2]. Upon annealing, the position of all the atoms in the network evolve giving rise to variations of the average network parameters such as reduction of the tetrahedral bond angle distortion and therefore of the average strain in the material. Great attention has been devoted to structural relaxation in ion implanted a-Si since there have been indications that this process occurs through annihilation of defects of the same kind as the ones observed in damaged crystalline material [3].

In this paper we present a detailed study of the implantation induced damage in Si by optical absorption measurements extending from energies above the band gap down to energies in the sub gap region of $\mathrm{Si}$, in order to obtain further informations on the investigated phenomena. The changes induced in the optical band gap, band edge slopes and in the sub gap features of the spectra are described. The various stages of formation and quenching of divacancies were monitored as a function of implantation conditions and 
annealing cycles. It is shown that the divacancies strongly affect the population of band tail states. Studies carried out in annealed damaged crystalline material reveal that the progressive annealing of the divacancy band is followed by the appearance of another absorption band, characteristic of some intrinsic secondary defect, whose annealing behaviour is similar to the one observed for the 5-vacancies EPR spectrum. Finally, the study of the structural relaxation process in implanted a-Si gives indications that the process is indeed associated with annihilation of defects as well as average strain reduction in the material.

\section{Experimental}

The investigations were performed on $800 \mathrm{~nm}$ thick [100] intrinsic Si on sapphire (SOS) layers in order to be able to measure absorption even in the spectral region above the band gap. The optical absorption measurements were performed by Photothermal Deflection Spectroscopy (PDS) thanks to which we were able to obtain data down to $0.5 \mathrm{eV}$ and therefore far into the subgap region of $\mathrm{Si}$. The interference induced oscillations which were observed as a function of energy in the PDS spectra was eliminated by performing the ratio of the simultaneously determined transmission to PDS spectra. An oscillation free resulting spectrum is obtained in this way. Moreover the resulting spectrum does not depend on eventual wavelength dependent reflectivity variations which can occur over the investigated spectral region. Absolute absorption values were obtained by normalising the spectra with respect to corresponding values obtained in the spectral region were the samples were optically opaque.

The implantation was carried out at 80 and $300 \mathrm{~K}$ and, on each sample, ions were implanted at 150 and $300 \mathrm{keV}$ (dose ratio $0.4: 1$ ) in order to obtain a fairly uniform relatively thick $(-500 \mathrm{~nm}$ ) implanted layer. Self ion irradiation was chosen to eliminate the effects arising from chemically dissimilar ions on the damage formation and evolution. The damage profile in the samples was determined by Rutherford Backscattering (RBS) and the structure of the implanted layers was monitored by Reflection High Energy Electron Diffraction (RHEED).

\section{Results and discussion}

\subsection{Effect of implantation conditions.}

Fig. 1 show the spectra relative to the samples implanted with increasing ion dose (dose values refer to the total dose implanted in each sample) implanted at $300 \mathrm{~K}$. Implantation at $80 \mathrm{~K}$ with a dose of $8.4 \times 10^{14}$

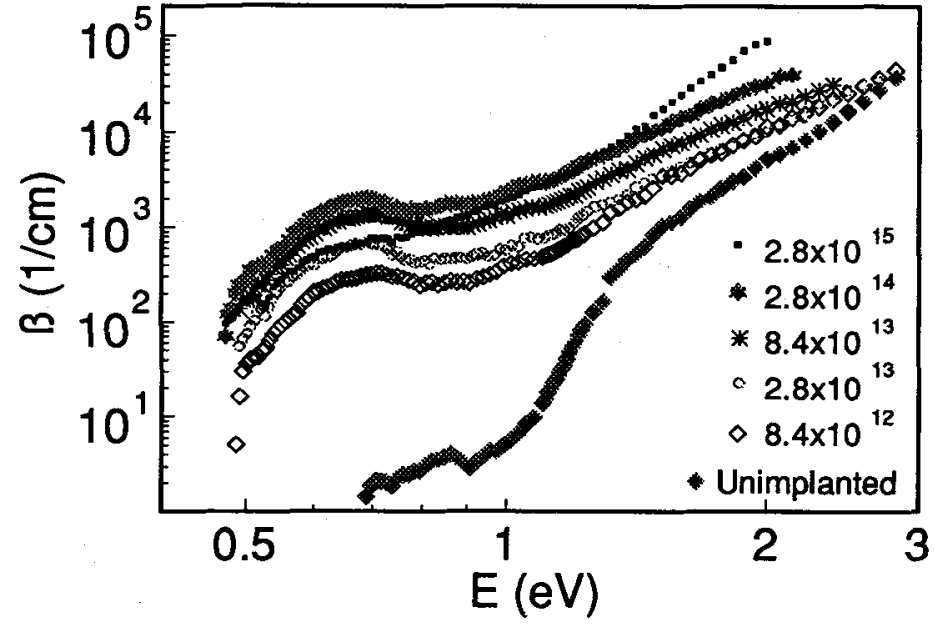

Fig. 1 Dose dependence of the absorption spectra of room temperature implanted $\mathrm{Si}$ on sapphire. $\mathrm{cm}^{-2}$ led to the formation of a uniform amorphous layer while the $300 \mathrm{~K}$ implantations led to the formation of non uniform damaged layers consisting of damaged crystalline material or of a mixture of amorphous and damaged crystalline material. The spectra show a progressive increase of the absorption with dose all over the investigated spectral region, due to the progressive accumulation of damage, as long as the implanted layer consisted of damaged crystalline material. When, with further dose increase, also amorphous material started forming in the layer, a decrease of the absorption in the spectra lower 


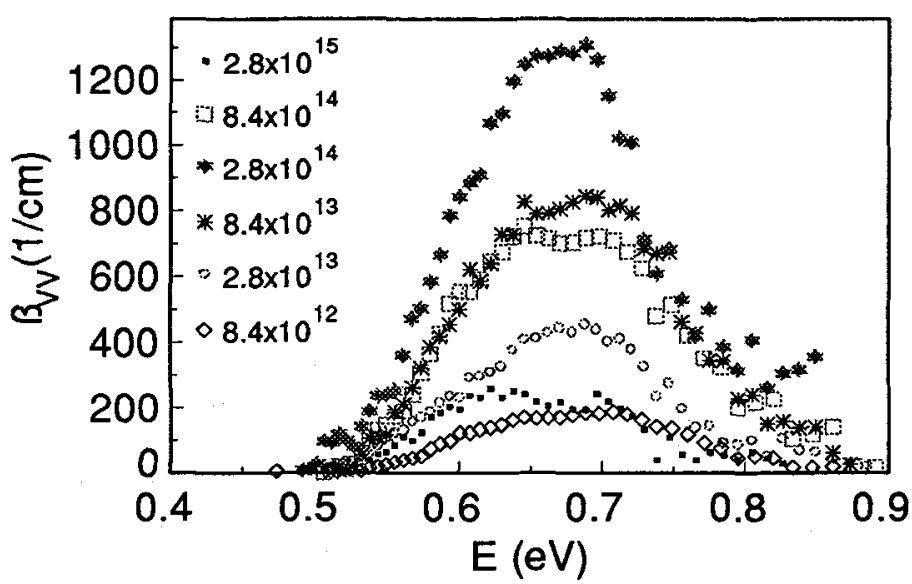

Fig.2 Dose dependence of the divacancy absorption band in room temperaturte implanted Si on sapphire.

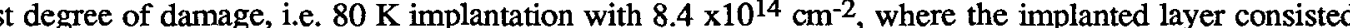
of amorphous material. From $\beta_{Y V}$ we have calculated the concentration of divacancies according to the relation $N_{W}\left(\mathrm{~cm}^{-3}\right)=7.7 \times 10^{16} \beta_{W}$ max , as suggested in ref. $5,\left(\beta_{W}\right.$ max is the maximum value of $\beta_{W}$ in the band ) and the average number of divacancies produced per ion $R_{V V}=N_{V V} / l \Phi$, where $\Phi$ is the ion dose and $l$ is the implanted layer thickness. Fig. 3 reports the dose dependence of such parameters. $N_{V V}$ initially increases, reaches a maximum value of $-10^{20} \mathrm{~cm}^{-3}$ at a dose of $2 \times 10^{14} \mathrm{~cm}^{-2}$ and then gradually becomes quenched for doses exceeding such a value. $R_{W}$, on the other hand, initially shows a slight decrease which then becomes substantial for doses exceeding $2 \times 10^{14} \mathrm{~cm}^{-2}$. Similar results have been previously obtained in bulk implanted $S i$ where the maximum attained value for $N_{V V}$ was $7 \times 10^{19} \mathrm{~cm}^{-3}[6]$.

The divacancy band quenching has been attributed to interactions amongst divacancies or interaction with other defects which anneal in parallel with the divacancies [6]. This is likely to occur for doses such that overlapping of the damage regions from the different ions becomes substantial, due to the considerable reduction induced in the average distance between the divacancies, and would cause the production rate of divacancies to become non-linear with respect to the implantation dose. A considerable reduction in the average number of divacancies produced per ion would then be expected, as we report in fig. 3 for doses

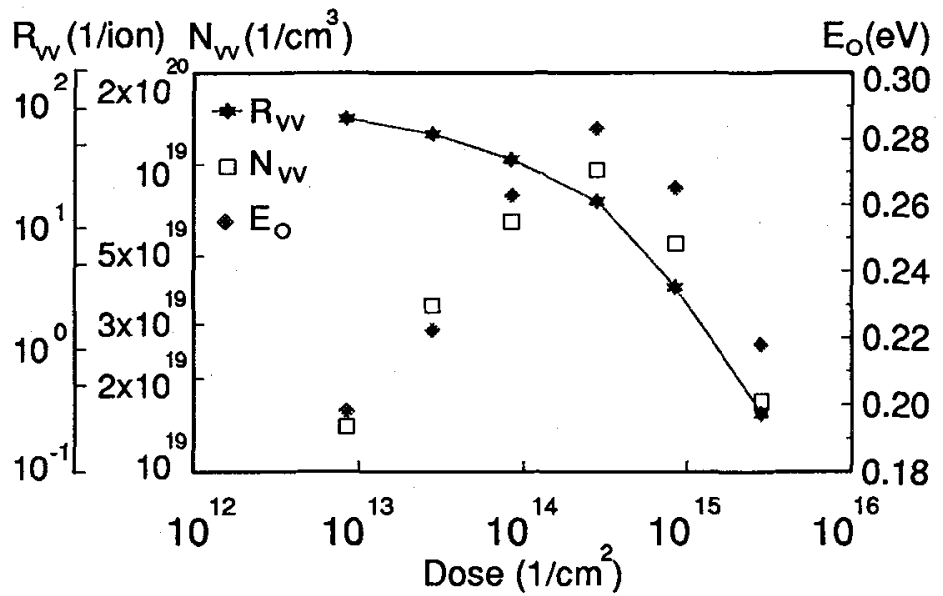

Fig.3 Dose dependence of $R_{V V}, N_{V V}$ and $E_{O}$ (see text) for room temperature implanted Si on sapphire. exceeding the one corresponding to the maximum divacancy concentration.

In fig. 3 we also report the dose dependence of the inverse logarithmic slope, $E_{O}$, which characterises the exponential dependence of the absorption in the band edge region, where the absorption depends on the distribution of band tail states. The $E_{o}$ values have been obtained by fitting the various absorption spectra in the respective band edge regions. The dose dependence of $E_{O}$ closely correlates with the one of $N_{W V}$ which suggests that the electronic states associated with the 


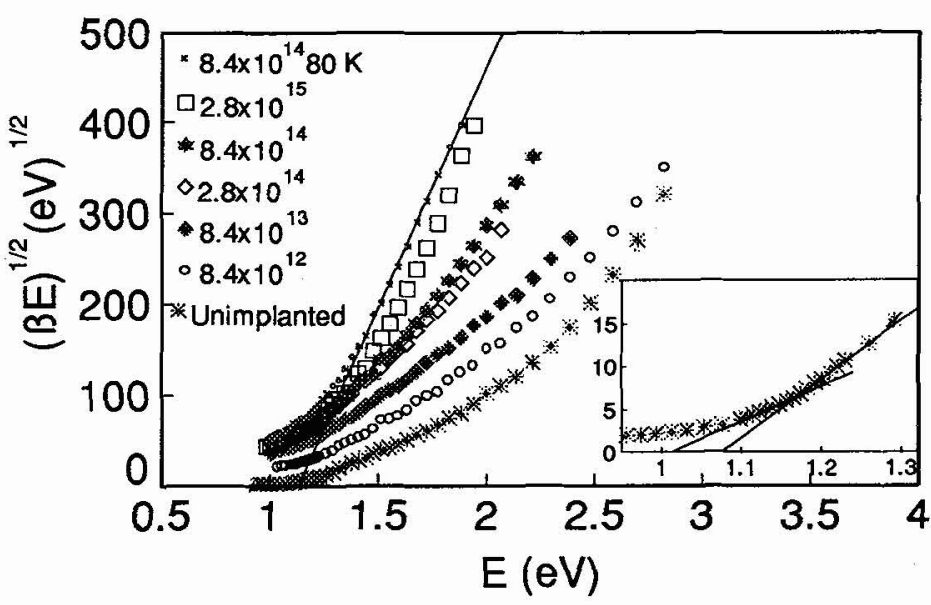

Fig. 4 Dose dependence of $(B E)^{1 / 2}$ vs $E$ plots for implanted Si on sapphire. Insert refers to crystalline material. divacancies strongly affect the population of band tail states.

In fig. 4 we report $(\beta E)^{1 / 2}$ vs $E$ in order to probe the effect of the implantation conditions on the value of the optical gap, $E_{G}$, and on the dependence of the absorption coefficient from $E_{G}$ near the band edge region. For unimplanted crystalline $\mathrm{Si}$, near the band edge region, the absorption is governed by phonon assisted transitions [7]. For low enough values of the absorption, where direct transitions are not involved, the $(\beta E)^{1 / 2}$ vs $E$ plot should yield a dual linear dependence as shown in the insert of fig. 4. The linear fits through the two linear dependent regions yield intercepts on the abscissa whose arithmetic mean provides the value of $E_{G}$ [7]. The value we obtain, $1.04 \pm 0.1 \mathrm{eV}$, is very close to the one obtained at $300 \mathrm{~K}, 1.08 \mathrm{eV}$, reported in ref. 8 for bulk crystalline Si. For amorphous material, on the other hand, for absorption values of the order of $10^{4} \mathrm{~cm}^{-1}$, the energy dependence of $\beta$ is given by the Tauc formula $\beta E=K\left(E-E_{G}\right)^{2}$, where $K$ is a constant, which yields a linear dependence in the plot of fig. 4 whose intercept on the abscissa provides $E_{G}$ for the fully amorphised layer for which we have obtained $E_{G}=1.15 \mathrm{eV}$. This value is larger than the one for the crystalline material but smaller than the ones obtained for other kinds of a-Si such as the ones obtained by room temperature sputtering $(1.26 \mathrm{eV})$ [9].

\subsection{Annealing of damaged crystalline material}

Fig. 5 shows the spectra relative to the sample implanted at $300 \mathrm{~K}$ with a dose of $2.8 \times 10^{14} \mathrm{~cm}^{-2}$, which

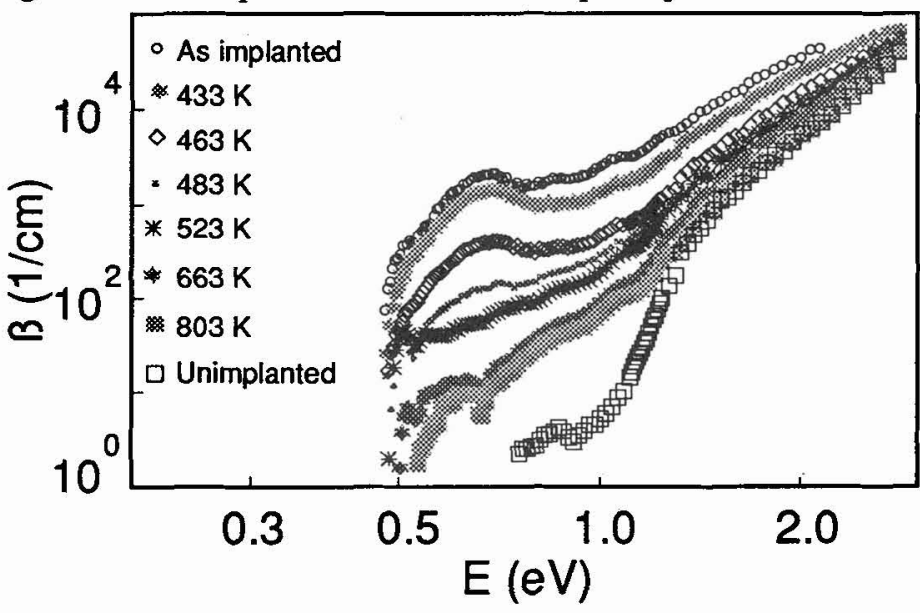

Fig.5 Annealing temperature dependence of the absorption spectra of SOS implanted at room temperature with $2.8 \times 10^{14} \mathrm{~cm}^{-2}$ Si ions. had shown the largest sub gap absorption associated with divacancies and other defects, following isochronal annealings subsequently carried out for 15 min. at increasing temperatures. The annealing temperatures was increased in steps of $40 \mathrm{~K}$ between 393 and $433 \mathrm{~K}, 30 \mathrm{~K}$ between 433 and $463 \mathrm{~K}, 20 \mathrm{~K}$ between 463 and $523 \mathrm{~K}$ and $50-70$ $\mathrm{K}$ between 523 and $803 \mathrm{~K}$. For the sake of clarity in the graph representation we only report spectra relative to some of the intermediate annealing stages. Nevertheless it is clear from the reported spectra that the absorption decreases all over the 


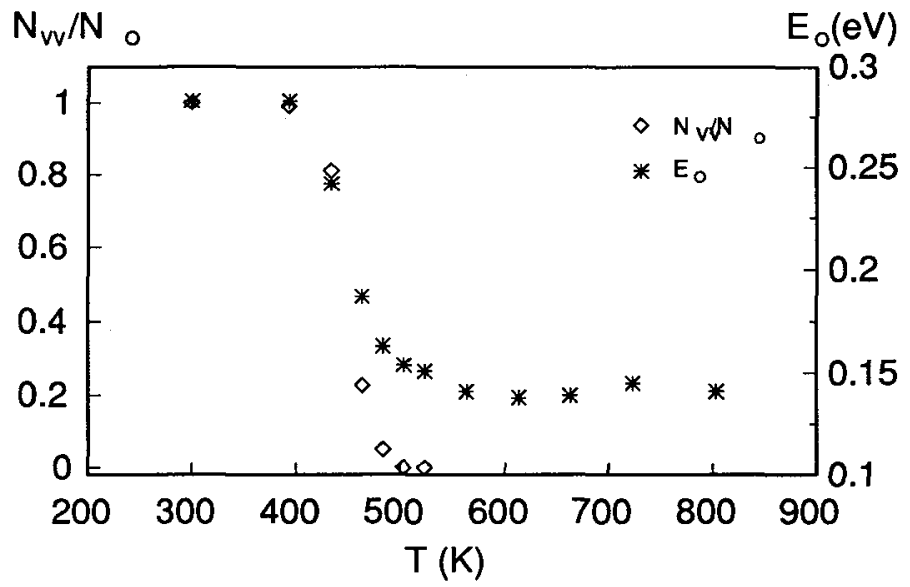

Fig. 6 Annealing temperature behaviour of relative $\mathrm{N}_{\mathrm{VV}}$ and of $\mathrm{E}_{\mathrm{O}}$ for SOS as in fig. 5 .

the band edge region is observed to progressively sharpen. Finally, intrinsic secondary defect, appears at $0.51 \mathrm{eV}(-2.45 \mu \mathrm{m})$ for annealing temperatures exceeding $463 \mathrm{~K}$ and could no longer be detected following annealing at $803 \mathrm{~K}$. These last three aspects are shown in greater details in fig. 6,7 and 8 respectively. In particular, fig. 6 shows that there is once more a strong correlation between the relative divacancy concentration and $E_{O}$. Once the divacancies anneal out, the $E_{O}$ value remains constant around $0.14 \mathrm{eV}$ up to the maximum annealing temperature reached in this work. This value is still considerably larger than the one we had obtained for unimplanted material $(0.07 \mathrm{eV})$ and is probably associated to residual point defects, point defects complexes, and strain induced by extended defects which survive up to much larger temperatures.

The annealing behaviour of the $0.51 \mathrm{eV}$ absorption band is shown in fig. 7. From the spectra, the absorption due to the band alone is obtained by subtracting the background absorption as previously described, and the band peak absorption, $\beta_{b}$, is reported in fig. 8 as a function of the annealing temperature. In an effort to find out the origin of such an absorption band, we have seeked, amongst the known intrinsic secondary defects, for one whose annealing behaviour resembled most the one of our band. In fig. 8 we have reported the comparison between the annealing behaviour of the $N$ centre [10], later identified as a 5vacancy complex [11], and the one of our band. A close correlation between the two behaviours is evident,

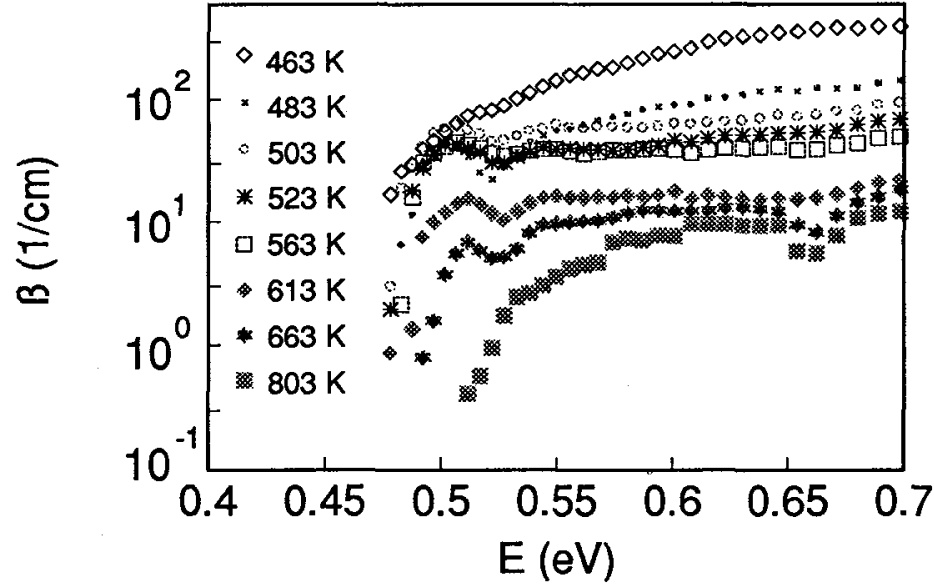
though certainly not yet conclusive.

\subsection{Annealing of amorphous material}

The study of the annealing on amorphous material, leading to the structural relaxation of ion implanted a-Si, was performed on the sample implanted with a dose of $8.4 \times 10^{14} \mathrm{~cm}^{-2}$ at $80 \mathrm{~K}$ which, as stated earlier on, consisted of a uniform layer of amorphous material.

Fig. 9 shows the spectra relative to the as implanted sample and to the sample after having undergone 15

Fig.7 Low energy region of spectra of fig. 5 . 


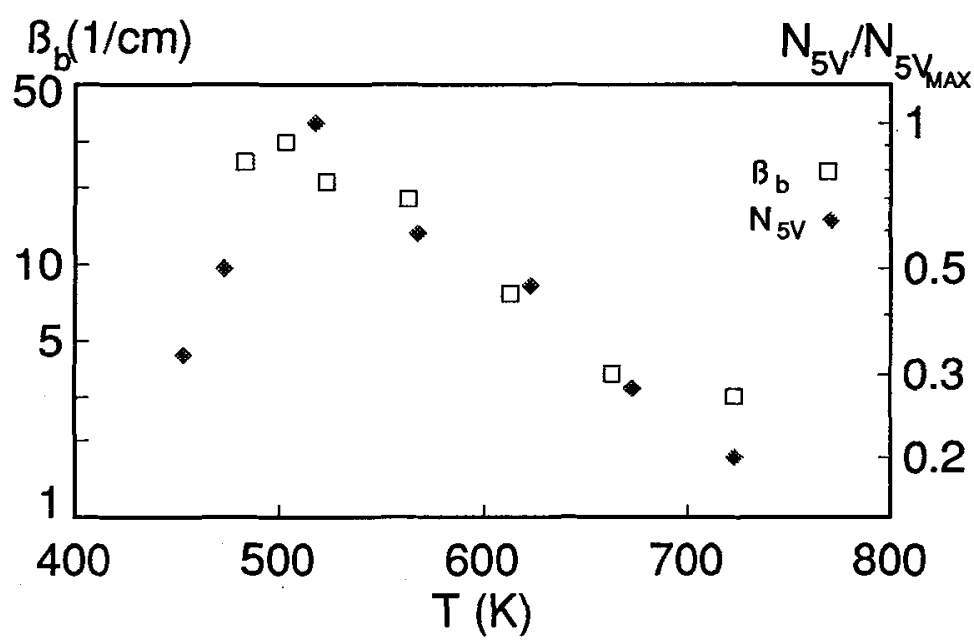

Fig.8 Annealing temperature dependence of $B_{b}$ (see text) for a sample processed as in fig. 5 and of 5-vacancy centres determined from EPR. min isochronal heat treatments, subsequently carried out at increasing temperatures, up to a maximum value of $793 \mathrm{~K}$. Also shown is the spectrum relative to an additional annealing at $793 \mathrm{~K}$ for 1 hour. Annealing for a total of 2 hours at $793 \mathrm{~K}$ did not produce any further changes in the spectrum which is therefore not reported. We note that, with the progressive increase in heat treatment, the absorption values decrease all over the investigated spectral region, while the absorption edge progressively shifts to larger energies and sharpens. The structural relaxation phenomenon thus leads to a continuous increase in

the optical gap of the material and to a decrease in the inverse logarithmic slope value as reported in fig. 10 which shows that the 1 hour annealing at $793 \mathrm{~K}$ leads to a saturation their values.

The progressive sharpening of the band edge which is observed with increasing heat treatments is to be ascribed to the progressive reduction of strain in the material which is known to affect the exponential absorption edge in semiconductors [12]. A reduction in strain, during the structural relaxation process in ion implanted a-Si, has in fact been detected by Raman spectroscopy measurements [6] and has also been related to the relaxation enthalpy detected by calorimetric measurements [6]. The progressive increase in $E_{G}$ is also associated to occurring strain reduction as reported for structural disorder associated results in aSi:H [12] where it has been observed that a reduction in thermally or structurally induced disorder in the material also leads to an increase of $E_{G}$ and a decrease of $E_{O}$. In particular, the authors in ref. 12 describe both $E_{G}$ and $E_{O}$ in terms of the mean square of the thermally and disorder induced displacement of the atoms from their equilibrium positions. They eventually obtain a linear dependence between the two

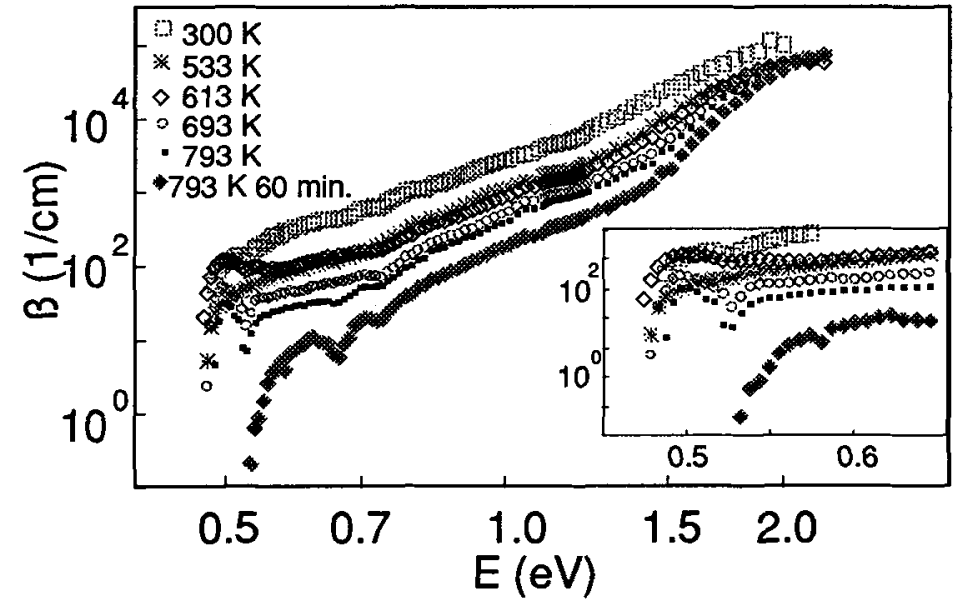

Fig.9 Annealing temperature dependence of absorption spectra of ion implanted a-Si layers. parameters which is supported by their experimental data and which is also obtained in the case of our ion implanted a-Si as reported in fig. 11. The results we obtain therefore also indicate the occurrence of strain reduction during the structural relaxation process.

Regarding the sub gap region of the spectra, the absorption in such a region depends on the defects present in the material. In a-Si:H the excess sub gap absorption with respect to the exponential band tail has enabled the determination of the absolute concentration of dangling bonds over a range of three orders of magnitude [13]. According to an 
optical sum rule such concentration is proportional to the integral of over the sub gap energy region. We

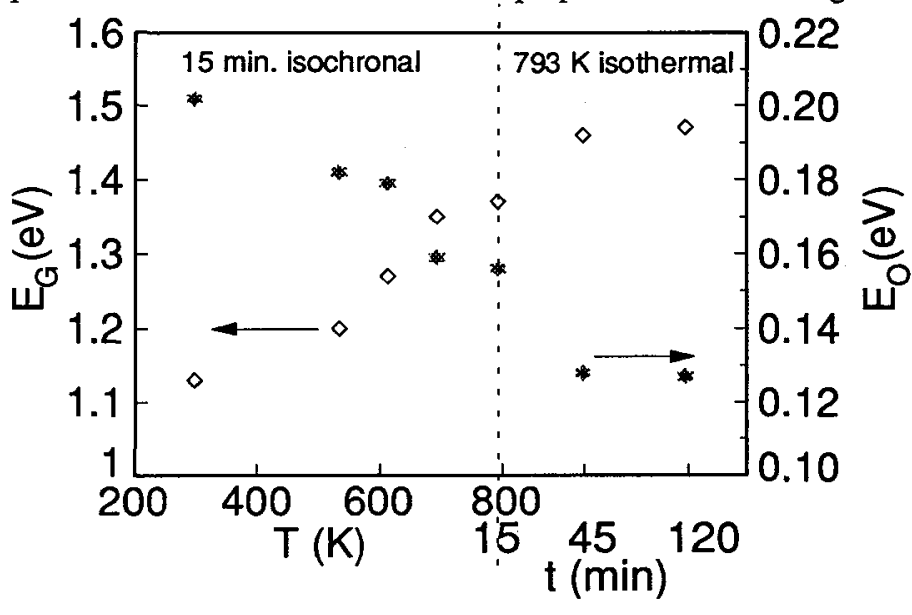

Fig. $10 \mathrm{E}_{\mathrm{G}}$ and $\mathrm{E}_{\mathrm{O}}$ vs annealing conditions for ion implnanted a-Si layer.

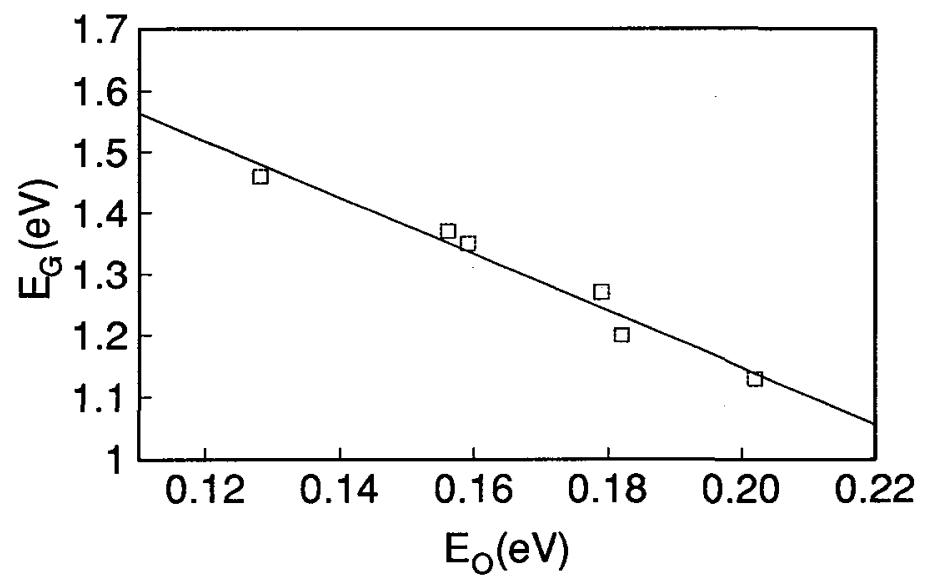

Fig.11 $E_{G}$ vs $E_{O}$ plot for annealed ion implanted a-Si have performed such integrals and we have found a reduction of nearly a factor 5 in the values calculated for the sample which had undergone maximum relaxation with respect to the one relative to the as implanted sample. The reduction is therefore greater than the factor 2 reported for the decrease in concentration of dangling bonds alone in relaxed ion implanted a-Si as determined by electron paramagnetic resonance [3] and almost the same as the one reported for the reduction of point defects concentration in relaxed material $(\sim$ factor 5$)$ as determined by metal diffusion and solubility measurements [3]. The results we have obtained therefore point in the same direction as earlier suggestions and that is that the structural relaxation process in ion implanted a-Si is accompanied by point defect annihilation. In this respect it is also interesting to point out that, even in these samples, an absorption band appears in the sub gap absorption spectra near $0.51 \mathrm{eV}$ (see insert of fig. 9) at the annealing temperature of $613 \mathrm{~K}$, gradually decreasing for larger temperatures and is no longer detectable when the sample becomes fully relaxed. Such an evolution should indicate the formation of secondary defect complexes during the intermediate stages of the annealing and therefore also point in the direction of defects evolution and annihilation during the structural relaxation process of ion implanted a-Si.

\section{Conclusions}

We have presented a detailed study of the implantation induced effects in $\mathrm{Si}$ on sapphire films through optical absorption measurements carried out form energies above the band gap down to energies far into the sub gap region of the material. The changes induced in the optical band gap, band edge region and sub gap features of the spectra have been carefully analysed as a function of the implantation and subsequent annealing conditions.

In damaged crystalline material, the various stages of formation and quenching of the divacancies were monitored through their $0.69 \mathrm{eV}(1.8 \mu \mathrm{m})$ absorption band. Correlation of the behaviours of such bands with that of the band edge slope as a function of implantation dose and annealing cycles have shown that divacancies strongly affect the population of band tail states. Moreover, the annealing studies revealed the 
progressive quenching of the divacancy band followed by the appearance of another absorption band, peaked at about $2.45 \mu \mathrm{m}$ and characteristic of some secondary intrinsic defect, whose annealing behaviour is very similar to the one observed for the EPR spectrum of the 5-vacancy complex.

Studies carried out on an amorphous ion implanted film as a function of annealing conditions allowed the investigation of the structural relaxation process in ion implanted a-Si. It has been observed that, in agreement with earlier observations, the relaxation process is associated with strain reduction in the material which leads to a progressive sharpening of the band edge and an increase of the optical gap of the material. Moreover, changes occurring upon relaxation of the material in the sub gap region of the spectra are consistent with earlier evidence that the relaxation process is accompanied by point defects and point defects complexes evolution and annihilation.

\section{Acknowledgements}

One of the authors, K.N.M., acknowledges financial support from I.C.T.P. Trieste. Research partially supported by Italian Ministry of Scientific Research and National Research Council.

\section{References}

1. H. Wong, N.W. Cheng, P.K. Chu, J. Liu and J.W. Mayer, Appl. Phys. Lett., 52, 1023 (1988).

2. D.E. Polk, J. Non Cryst. Solids, 5, 365 (1971).

3. See for example: S. Roorda, W.C. Sinke, J.M. Poate, D.C. Jacobson, S. Dierker, B.S. Dennis, D. J. Eaglesham, F. Spaepen and F. Fuoss, Phys. Rev. B, 44, 3702 (1991).

4. L. J. Cheng, J.C. Corelli, J.W. Corbett and G.D. Watkins, Phys. Rev., 152, 761 (1966).

5. L.J. Cheng and J. Lori, Phys. Rev., 171, 856 (1968).

6. H.J. Stein, F.L.Vook, D.K. Brice, J.A. Borders and S.T. Picraux, Rad. Effects, 6, 19, (1970).

7. N.F. Mott and E.A. Davies in "Electronic Processes in Non Crystalline Materials" (Clarendon Press Oxford 1979), p. 274.

8. G.G. Mac Farlane and V. Roberts, Phys. Rev., 98, 1865 (1955).

9. A. Lewis, Phys. Rev. Lett. 29, 1555 (1972).

10. W. Jung and G. S. Newell, Phys. Rev., 2, 648 (1963).

11. Y.H. Lee and J.W. Corbett, Phys. Rev. B, 8, 2810 (1973).

12. G.D. Cody, T. Tiedje, B. Abeles and Y. Goldstein, Phys. Rev. Lett. 47, 1480 (1981).

13. N. Amer and J.D. Jackson, in "Semiconductors and Semimetals", Vol 21B, edited by J.I. Pankove (Academic Press, New York, 1984) p. 83. 